\title{
Clinical value of homodynamic numerical simulation applied in the treatment of cerebral aneurysm
}

\author{
HAILIN ZHANG ${ }^{1}$, LI LI ${ }^{2}$, CHONGJIE CHENG $^{1}$ and XIAOCHUAN SUN ${ }^{1}$ \\ ${ }^{1}$ Department of Neurosurgery, The First Affiliated Hospital of Chongqing Medical University, Chongqing 400016; \\ ${ }^{2}$ School of Clinical Medicine, Gansu University of Chinese Medicine, Lanzhou, Gansu 730030, P.R. China
}

Received May 23, 2017; Accepted September 12, 2017

DOI: 10.3892/etm.2017.5189

\begin{abstract}
Our objective was to evaluate the clinical value of numerical simulation in diagnosing cerebral aneurysm based on the analysis of numerical simulation of hemodynamic model. The experimental method used was the numerical model of cerebral aneurysm hemodynamic, and the numerical value of blood flow at each point was analyzed. The results showed that, the wall shear stress (WSS) value on the top of CA1 was significantly lower than that of the top $(\mathrm{P}<0.05)$, the WSS value of each point on the CA 2 tumor was significantly lower than that of tumor neck $(\mathrm{P}<0.05)$; the pressure value on the tumor top and tumor neck between CA1 and CA 2 had no significant difference $(\mathrm{P}>0.05)$; the unsteady index of shear (UIS) value at the points of 20 had distinctly changed, the wave range was 0.6-1.5; the unsteady index of pressure value of every point was significantly lower than UIS value, the wave range was $0.25-0.40$. In conclusion, the application of cerebral aneurysm hemodynamic research can help doctors to diagnose cerebral aneurysm more precisely and to grasp the opportunity of treatment during the formulating of the treatment strategies.
\end{abstract}

\section{Introduction}

Related research showed that cerebral aneurysm is one of the most common malignant tumors at the present stage in clinic, the specific mechanism of the occurrence and development of cerebral aneurysm is still not clear (1). However, most scholars considered that there was a close correlation between hemodynamic abnormality and the occurrence and development of aneurysms or angiorrhexis (2). With the development and progress of science and technology, research including animal experiments, clinical imaging observation, in vitro model

Correspondence to: Dr Xiaochuan Sun, Department of Neurosurgery, The First Affiliated Hospital of Chongqing Medical University, 1 Youyi Road, Yuzhong, Chongqing 400016, P.R. China E-mail: ryp3ay@163.com

Key words: hemodynamic, numerical simulation, clinical value, cerebral aneurysm study all have played a very important role during the study of cerebral aneurysm hemodynamic (3). Research of cerebral aneurysm hemodynamic has gradually become the research emphasis at the present stage along with the rapid advances of computer technology and the improvement of computer operational performance and calculation software (4). The study of hemodynamic has good controllability and a high economic advantage in general, so it has wide application (5). Thus, we decided to assess the clinical value of numerical simulation in diagnosing cerebral aneurysm based on the analysis of numerical simulation of hemodynamic model.

\section{Materials and methods}

Model reconstruction. In this study, three-dimensional spiral angiography machine (Phillips, New York, NY, USA) was used to collect the DSA image, and the image on the three-dimensional angiography workstation was reconstructed, we chose suitable windows, intercepted the peripheral artery of cerebral aneurysm and branch, then read image data with Geomagic Studio 9.0 (Geomagic, Morrisville, NC, USA), for the data file.

Coordinates extraction. MATLAB6.5 software (MathWorks, Natick, MA, USA) was used to create and import the bmp files, write the bmp path of monochromatic aneurysm into data mat, and to obtain the two-dimensional coordinates of every points. Coordinate point value was copied and was saved in txt file as data formatted text file.

Mesh generation. Gambit software (Ansys, Pittsburgh, PA, USA) was used to create and import the point coordinates file, reproduce the tumor contour of every point, connect every point with lines, confirm the boundary contour, mark the region of interest, such as tumor wall, and the tumor neck.

Boundary definition. The velocity inlet boundary and flow exit boundary were set of as two entrance boundaries, solid wall boundary was set as another boundary. Flow exit boundary and velocity inlet boundary were set of Para tumor, other settings were like the boundary setting of apical aneurysm boundary.

Mesh dividing. All the contour images were changed into a plane, the plane was dived by two-dimensional mesh, and 

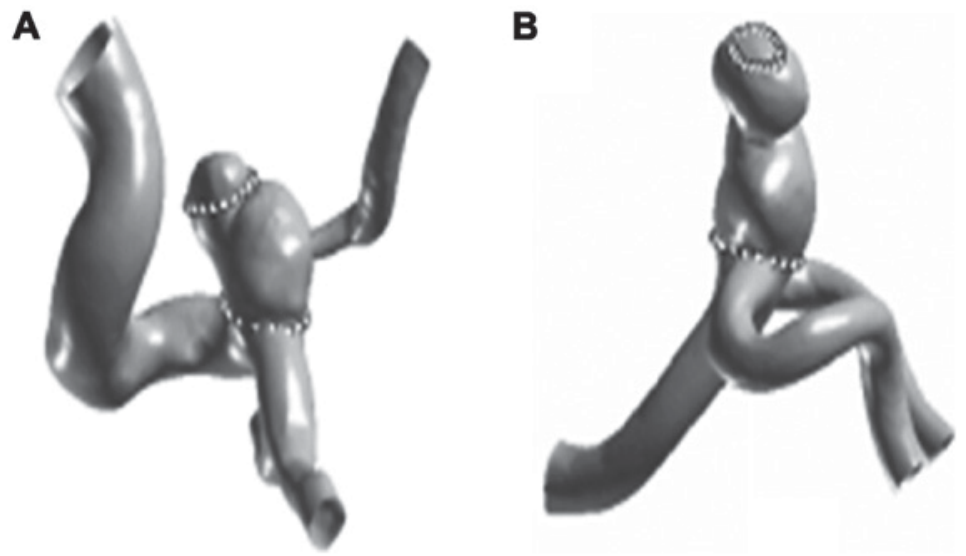

Figure 1. The top and neck of cerebral aneurysms. (A) CA1 take point map; (B) CA2 point drawing.
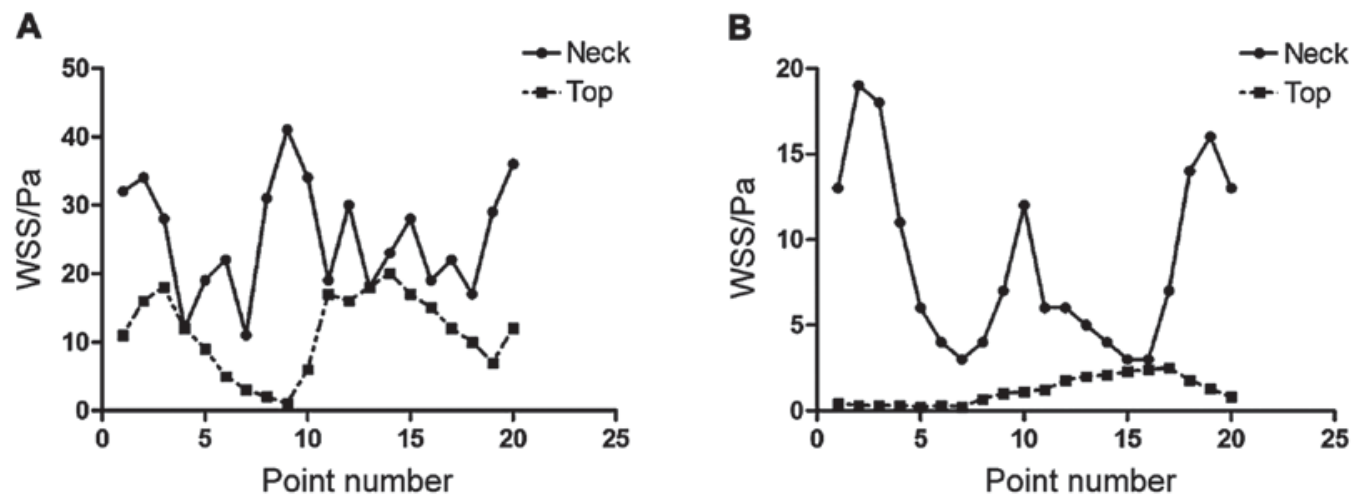

Figure 2. Calculation results of WSS value at each point. (A) CA1 tumor neck and top of each point WSS value; (B) CA1 tumor neck and top of each point WSS value. WSS, wall shear stress.

saved as mesh format. Then the lateral aneurysm plan image was opened with Gambit software, split aneurysm and blood vessels with virtual connection line, and continued to divide two-dimensional mesh, the number of units which were divided of CA1 and CA2 were 1507070 and 874595.

Blood flow behavior simulation. The Fluent software (Ansys) was run to import the msh file of aneurysm, to set tumor boundary conditions and assumptions, to calculate the blood flow. Tube diameter/tube diameter length $=$ scale factor, actual velocity of single tumor was the blood flow speed. First, the viscous parameter of flowing blood in the vessel $(0.002 \mathrm{~Pa} / \mathrm{sec})$ and the density of flowing blood in the vessel $\left(1,059 \mathrm{~kg} / \mathrm{m}^{3}\right)$ were set, then a cardiac cycle iteration was completed, pressure, velocity, shear stress time curve were found, selection of the point of interest, was chosen for every point according to the curvilinear trend, and then analyzed.

\section{Results}

Results of the points. In this study, 20 points were taken on the top and neck of cerebral aneurysm, the wall shear stress (WSS) and total pressure of every point during systolic time were drawn respectively, as shown in Fig. 1.

WSS results of each point. The results of this study showed that the WSS value on the top of CA1 was significantly lower than the WSS value on the top $(\mathrm{P}<0.05)$, the WSS value of every points on the tumor top of CA2 were significantly lower than the WSS value of tumor neck $(\mathrm{P}<0.05)$, and the WSS value of tumor neck has more heterogeneous character than the WSS value of tumor top, as shown in Fig. 2.

Pressure value calculating results of each point. The results of this study showed that there was no significant difference between the pressure of tumor neck (CA1 and CA2) and the pressure on the tumor top $(\mathrm{P}>0.05)$, as shown in Fig. 3.

UIS and UIP calculation of results of each point. The fluctuation of WSS and pressure caused by the change of time were quantitative analyzed by unsteady index of shear (UIS) and unsteady index of pressure (UIP). The results showed that there has significant difference of UIS value changes of these 20 points, $0.6-1.5$ was the wave range. The UIP value of each point was significantly lower than the UIS value, $0.25-0.40$ was the wave range, as shown in Fig. 4.

\section{Discussion}

Related research showed that the main reason which caused the occurrence of aneurysm were elastic friction in arterial wall and destruction, relaxation, defect of smooth muscle layer (6). The extra vascular elastic membrane (EEM) plays a vital role in maintaining the elasticity, length, bending degree of arterial 
A

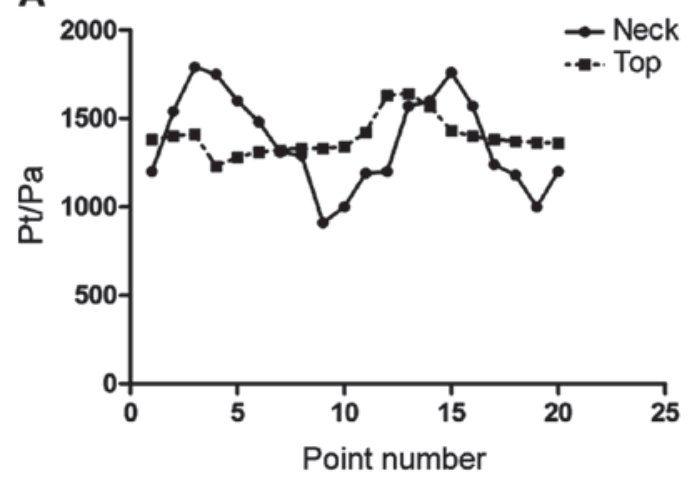

B

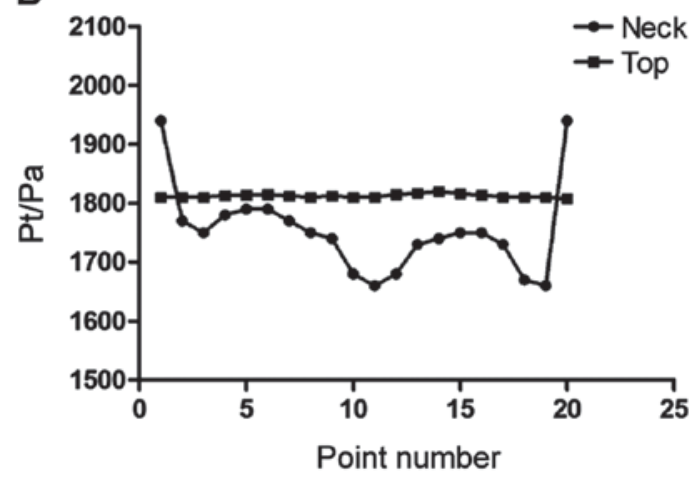

Figure 3. Calculation results of pressure values at each point. (A) CA1 tumor neck and top pressure value of each point; (B) CA1 tumor neck and the top of the pressure value of each point.

A

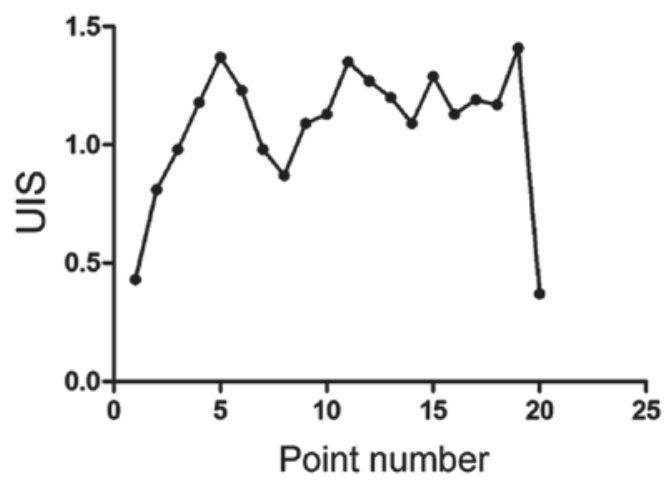

B

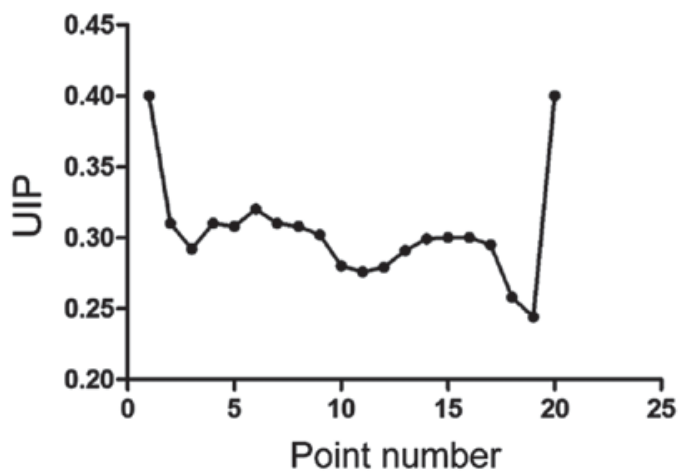

Figure 4. UIS and UIP values at each pointon the top of the CA2 tumor. (A) CA2 tumor at the top of each point UIS; (B) CA2 tumor top points UIP. UIS, unsteady index of shear; UIP, unsteady index of pressure.

wall (7). Jain et al injected elastase into adventitial coat of abdominal aorta, spindle aneurysm can be seen in $24 \mathrm{~h}$, and the dissolving of middle-level elastic lamina, endomembrane tissue injury and the disorganization of smooth muscular tissue can also be seen, they reached the peak in the third day, and the aortic aneurysm is significantly shrunken after 42 days (8). Some scholars pointed out that the appearance of irreversible damage of smooth muscle cells may cause the formation of aneurysm, and medial smooth muscle may be more important than elastic film, elastase can help smooth muscle cells to be regenerated (9). The occurrence of vascular abnormalities is related to protein, cytokines, apoptosis and inflammation. The extracellular matrix (ECM) of artery includes reticular fibers and collagen fibers, elastic fibers, noncollagenous glycoproteins, it can maintain the tension and elasticity of the arterial wall effectively, and can also maintain fixed shape under the impact of blood. Related research results showed that, total collagen protein on the tumor wall of patients with intracranial aneurysm reduced significantly compared with total cerebral aneurysms (10). A large amount of collagen can be seen distributing between adventitia of normal cerebral artery and tunicae media, but collagen distribution trends to diffuse on aneurysm wall, the immunofluorescence staining experiment result showed that the immunofluorescence staining intensity of collagen on aneurysm wall significantly abated. In normal cerebral artery wall, the expression of fibronectin (FN) was strongest in endothelial basement membrane (BM), and the expression of FN can be seen both in endothelial basement adventitial membrane and tunicae media membrane, but there was almost no expression of FN on the wall of human intracranial aneurysm (11). In normal cerebral arteries, type IV collagen is mainly distributed in subendothelial BM, it can be seen with low expression around smooth muscle cell in media tunicae, the distribution of type IV collagen were destroyed on the wall of tumor, the expression of type IV collagen debated, distribution dispersion can be seen, all of these caused significant reduction of type IV collagen expression in original endothelium. Hemodynamics may also be an important factor which caused the occurrence and development of cerebral aneurysm, some researchers analyzed the rat model and found that on side ligaturing the cephalic artery can cause renal hypertension of rats, the occurrence of cystic aneurysm, spindle aneurysm can be seen after ligaturing the cephalic artery 12 months later, so we can conclude that the change of homodynamic has close correlation with the occurrence of aneurysm (10-12). Research has found that the pressure, shear stress and tensile stress all were arterial wall stress. In-depth research is required to clarify how the above stress changed into biological signal when arterial wall was affected by mechanical signal $(13,14)$. In general, endothelial cells can change into biochemical reaction and electrophysiology when they meet mechanical force, leading to genetic expression changes and biological change. Some 
researchers pointed out that hemodynamic changes were the primary factors which caused the occurrence and development of aneurysml (12-14). It has been shown that internal pressure of aneurysm mainly act on the tumor wall and then cause the occurrence of stretch stress, and aneurysmal radius and intra tumor pressure all were positively correlated with stretch stress, stretch stress presented negative correlation with tumor wall thickness (15). Some researchers found that the stress in tumor wall was 10 times higher than normal level (16). Along with the growth of aneurysm, aneurysm tumor intramural degenerative change was always accompanied by the increasing of tumor radius, the reducing of tumor wall thickness, causing high intratumoral pressure and the occurrence of progressive tensile stress. At the present stage, the research on hemodynamics mainly include pathological vector model analysis and the analysis of inner flow field of artery with aneurysm artery, the analysis of inner flow field of artery with aneurysm artery can be studied according hydrodynamic to analyze the character of hemodynamics, seek for the relations between hemodynamics and pressure distribution, velocity distribution, wall shear force, this process has two parts: Numerical calculation and in vitro mode test. Now, with the rapid development of computer technology and imaging technology, the research on numerical simulation of 3D imaging in clinic has increased. Related research showed, the model has realistic geometry structure, can make a bridge between clinic and experimental research $(2,14-17)$. It was pointed out that the growth and break of cerebral aneurysm had close correlation with the distribution of WSS and pressure (2,15-18). In general, low WSS can cause aneurysm rupture, high WSS cause the growth of aneurysm. The results of this research showed that the WSS maximum value can be found in the position of vascular bifurcation and tumor neck, and the WSS minimum value can be found on the top of aneurysm, so the position with daughter tumor of aneurysm will rupture easily, all the studies above were consistent with the results of this study. Some researchers pointed out that the growth and break of aneurysm had close correlation with the change of pressure and shearing stress in vascular wall, so we investigated the fluctuation of pressure and WSS with time using UIS and UIP. The results of this research showed that the change regulation of UIS, UP or WSS were not always in the same manner, the results suggested that further research to analyze the relativity of aneurysm's growth and break and UIS, UIP and WSS.

In conclusion, the research of cerebral aneurysm hemodynamic can help doctors to judge the risk of aneurysm more accurate, and help to grasp the opportunity of treatment and developing treatment strategies.

\section{References}

1. Campodeaño L, Oliveira MSN and Pinho FT: A review of computational hemodynamics in middle cerebral aneurysms and rheological models for blood flow. Appl Mech Rev 67: 030801, 2015.

2. Futami K, Nambu I, Kitabayashi T, Sano H, Misaki K, Uchiyama $\mathrm{N}$ and Nakada M: Inflow hemodynamics evaluated by using four-dimensional flow magnetic resonance imaging and the size ratio of unruptured cerebral aneurysms. Neuroradiology 59: 411-418, 2017.
3. Mizuma A, Ishikawa T, Kajihara N, Takano H, Endo K, Kawakata M, Shibukawa S, Nakamura T, Nishio H, Horie T, et al: Dynamic cross-sectional changes of the middle cerebral artery in atherosclerotic stenosis detected by $3 \cdot 0$-Tesla MRI. Neurol Res 36: 795-799, 2014.

4. Sarrami-Foroushani A, Villa-Uriol MC, Nasr Esfahany M, Coley SC, Di Marco LY, Frangi AF and Marzo A: Modeling of the acute effects of primary hypertension and hypotension on the hemodynamics of intracranial aneurysms. Ann Biomed Eng 43: 207-221, 2015.

5. Zhang Y, Jing LK and Zhang QQ: Analysis of morphological and hemodynamic characteristics of unruptured posterior communicating artery aneurysms with oculomotor nerve paralysis. Chin J Neurosurg 32: 604-606, 2016.

6. Ivanov D, Dol A and Polienko A: Patient-specific hemodynamics and stress-strain state of cerebral aneurysms. Acta Bioeng Biomech 18: 9-17, 2016.

7. Krings T, Piske RL and Lasjaunias PL: Intracranial arterial aneurysm vasculopathies: Targeting the outer vessel wall. Neuroradiology 47: 931-937, 2005.

8. Jain K, Jiang J, Strother C and Mardal KA: Transitional hemodynamics in intracranial aneurysms - Comparative velocity investigations with high resolution lattice Boltzmann simulations, normal resolution ANSYS simulations, and MR imaging. Med Phys 43: 6186-6198, 2016.

9. Frolov SV, Sindeev SV, Potlov AY and Liepsch D: Numerical modeling of the effects of a flow-diverting stent on hemodynamic characteristics in a cerebral aneurysm. Biomed Eng (NY) 50: 363-366, 2017.

10. Turek G, Lewszuk A, Kochanowicz J, Zielińska-Turek J, Wilk P and Mariak Z: Evaluation of the effectiveness of endovascular embolization for the treatment of ruptured cerebral aneurysms. Pol Merkur Lekarski 42: 76-80, 2017 (In Polish).

11. Qian Z, Feng X, Kang H, Wen X, Xu W, Li Y, Jiang C, Wu Z and Liu A: Dissecting aneurysms of the distal segment of the posterior cerebral artery: Clinical presentation and endovascular management. Chin Neurosurg J 3: 7, 2017 (In Chinese).

12. Navratil O, Duris K, Juran V, Neuman E, Svoboda K and Smrcka M: Middle cerebral artery aneurysms with intracerebral hematoma-the impact of side and volume on final outcome. Acta Neurochir (Wien) 159: 543-547, 2017.

13. Zhang Y and Yang XJ: Advances in the study of hemodynamics of stent and coil embolization in the treatment of intracranial aneurysms. Chin J Cerebrovasc Dis 13: 372-375, 2016.

14. Saho T and Onishi H: Quantitative comparison of hemodynamics in simulated and 3D angiography models of cerebral aneurysms by use of computational fluid dynamics. Radiological Phys Technol 8: 258-265, 2015.

15. Wang C, Tian Z, Liu J, Jing L, Paliwal N, Wang S, Zhang Y, Xiang J, Siddiqui AH, Meng H, et al: Flow diverter effect of LVIS stent on cerebral aneurysm hemodynamics: A comparison with Enterprise stents and the Pipeline device. J Transl Med 14: 199, 2016

16. Turjman AS, Turjman F and Edelman ER: The role of fluid dynamics and inflammation in intracranial aneurysm formation. Circulation 129: 373-382, 2014.

17. Huang C, Shi B and Chai ZGZ: Multi-GPU based Lattice Boltzmann method for hemodynamic simulation in patientspecific cerebral aneurysm. Commun Comput Phys 17: 960-974, 2015.

18. Nair P, Chong BW, Indahlastari A, Ryan J, Workman C, Haithem Babiker M, Yadollahi Farsani H, Baccin CE and Frakes D: Hemodynamic characterization of geometric cerebral aneurysm templates treated with embolic coils. J Biomech Eng 138: 021011, 2016. 